CUBO A Mathematical Journal Vol.17, No 02, (31-48). June 2015

\title{
Refinements of the Generalized Trapezoid Inequality in Terms of the Cumulative Variation and Applications
}

\author{
S.S. DRAGOMIR ${ }^{1,2}$ \\ ${ }^{1}$ Mathematics, College of Engineering \\ ${ }^{2}$ School of Computational \\ $\&$ Science \\ \& Applied Mathematics, \\ Victoria University, PO Box 14428, \\ University of the Witwatersrand, \\ Melbourne City, MC 8001, Australia. \\ Private Bag 3, Johannesburg 2050, South \\ Africa, \\ sever.dragomir@vu.edu.au
}

\begin{abstract}
Refinements of the generalized trapezoid inequality for functions of bounded variation in terms of the cumulative variation function are given. Applications for selfadjoint operators on complex Hilbert spaces are also provided.
\end{abstract}

\section{RESUMEN}

Damos refinamientos de la desigualdad trapezoidal generalizada para funciones de variación acotada en términos de la función de variación acumulativa. También probamos aplicaciones a operadores autoadjuntos en espacios de Hilbert complejos.

Keywords and Phrases: Generalized trapezoid inequality, Functions of bounded variation, Cumulative variation, Selfadjoint operators

2010 AMS Mathematics Subject Classification: 26D15, 47A63. 


\section{Introduction}

The following generalized trapezoidal inequality was obtained in 1999 by the author [11, Proposition 1]

$$
\begin{aligned}
& \left|\int_{a}^{b} f(t) d t-(x-a) f(a)-(b-x) f(b)\right| \\
& \leq\left[\frac{1}{2}(b-a)+\left|x-\frac{a+b}{2}\right|\right] \bigvee_{a}^{b}(f),
\end{aligned}
$$

where $x \in[a, b]$. The constant $\frac{1}{2}$ cannot be replaced by a smaller quantity. See also [9] for a different proof and other details.

The best inequality one can derive from (1.1) is the trapezoid inequality

$$
\left|\int_{a}^{b} f(t) d t-\frac{f(a)+f(b)}{2}(b-a)\right| \leq \frac{1}{2}(b-a) \bigvee_{a}^{b}(f) .
$$

Here the constant $\frac{1}{2}$ is also best possible.

For related results, see [1-44, 6]-8], 10, [14, 15, 17, [18, 20], 22]-27] and 29- 32 .

The main aim of the present paper is to provide some refinements of the inequalities (1.1) and (1.2) in terms of the cumulative variation function. Applications for selfadjoint operators on complex Hilbert spaces are also given.

\section{Refinements of the Generalized Trapezoid Inequality}

For a function of bounded variation $v:[a, b] \rightarrow \mathbb{C}$ we define the Cumulative Variation Function $(\mathrm{CVF}) \vee:[a, b] \rightarrow[0, \infty)$ by

$$
V(t):=\bigvee_{a}^{t}(v)
$$

the total variation of $v$ on the interval $[a, t]$ with $t \in[a, b]$.

It is know that the CVF is monotonic nondecreasing on $[a, b]$ and is continuous in a point $c \in[a, b]$ if and only if the generating function $v$ is continuing in that point. If $v$ is Lipschitzian with the constant $\mathrm{L}>0$, i.e.

$$
|v(t)-v(s)| \leq L|t-s| \text { for any } t, s \in[a, b]
$$

then $\mathrm{V}$ is also Lipschitzian with the same constant.

The following lemma is of interest in itself as well, see also [16]. For the sake of completeness, we give here a simple proof. 
Lemma 2.1. Let $\mathrm{f}, \mathrm{u}:[\mathrm{a}, \mathrm{b}] \rightarrow \mathbb{C}$. If $\mathrm{f}$ is continuous on $[\mathrm{a}, \mathrm{b}]$ and $\mathrm{u}$ is of bounded variation on $[\mathrm{a}, \mathrm{b}]$, then

$$
\left|\int_{a}^{b} f(t) d u(t)\right| \leq \int_{a}^{b}|f(t)| d\left(\bigvee_{a}^{t}(u)\right) \leq \max _{t \in[a, b]}|f(t)| \bigvee_{a}^{b}(u)
$$

Proof. Since the Riemann-Stieltjes integral $\int_{a}^{b} f(t) d u(t)$ exists, then for any division

$$
I_{n}: a=t_{0}<t_{1}<\cdots<t_{n-1}<t_{n}=b
$$

with the norm

$$
v\left(I_{n}\right):=\max _{i \in\{0, \ldots, n-1\}}\left(t_{i+1}-t_{i}\right) \rightarrow 0
$$

and for any intermediate points $\xi_{i} \in\left[t_{i}, t_{i+1}\right], i \in\{0, \ldots, n-1\}$ we have:

$$
\begin{aligned}
\left|\int_{a}^{b} f(t) d u(t)\right| & =\left|\lim _{v\left(I_{n}\right) \rightarrow 0} \sum_{i=0}^{n-1} f\left(\xi_{i}\right)\left[u\left(t_{i+1}\right)-u\left(t_{i}\right)\right]\right| \\
& \leq \lim _{v\left(I_{n}\right) \rightarrow 0} \sum_{i=0}^{n-1}\left|f\left(\xi_{i}\right)\right|\left|u\left(t_{i+1}\right)-u\left(t_{i}\right)\right| .
\end{aligned}
$$

However,

$$
\left|\mathfrak{u}\left(t_{i+1}\right)-\mathfrak{u}\left(t_{i}\right)\right| \leq \bigvee_{t_{i}}^{t_{i+1}}(u)=\bigvee_{a}^{t_{i+1}}(u)-\bigvee_{a}^{t_{i}}(u),
$$

for any $i \in\{0, \ldots, n-1\}$, and by (2.2) we have

$$
\begin{aligned}
\left|\int_{a}^{b} f(t) d u(t)\right| & \leq \lim _{v\left(I_{n}\right) \rightarrow 0} \sum_{i=0}^{n-1}\left|f\left(\xi_{i}\right)\right|\left[\bigvee_{a}^{t_{i}+1}(u)-\bigvee_{a}^{t_{i}}(u)\right] \\
& =\int_{a}^{b}|f(t)| d\left(\bigvee_{a}^{t}(u)\right)
\end{aligned}
$$

and the last Riemann-Stieltjes integral exists since $|f|$ is continuous and $\bigvee_{a}$ is monotonic nondecreasing on $[a, b]$.

The last part follows from :

$$
\left|\int_{a}^{b} g(t) d v(t)\right| \leq \max _{t \in[a, b]}|g(t)|[v(b)-v(a)],
$$

holding for any function $\mathrm{g}$ continuous on $[\mathrm{a}, \mathrm{b}]$ and $v$ monotonic nondecreasing on $[\mathrm{a}, \mathrm{b}]$.

The details are omitted.

The following result may be stated.

Theorem 2.2. Let $\mathrm{f}:[\mathrm{a}, \mathrm{b}] \rightarrow \mathbb{C}$ be a function of bounded variation on $[\mathrm{a}, \mathrm{b}]$. Then 


$$
\begin{aligned}
& \left|\int_{a}^{b} f(t) d t-[f(a)(x-a)+f(b)(b-x)]\right| \\
& \leq \int_{a}^{x}\left(\bigvee_{a}^{t}(f)\right) d t+\int_{x}^{b}\left(\bigvee_{t}^{b}(f)\right) d t \\
& \leq(x-a) \bigvee_{a}^{x}(f)+(b-x) \bigvee_{x}(f) \\
& \leq\left\{\begin{array}{c}
{\left[\frac{1}{2}(b-a)+\left|x-\frac{a+b}{2}\right|\right] \bigvee_{a}^{b}(f),} \\
{\left[\frac{1}{2} \bigvee_{a}^{b}(f)+\frac{1}{2}\left|\bigvee_{a}^{x}(f)-\bigvee_{x}^{b}(f)\right|\right](b-a),}
\end{array}\right.
\end{aligned}
$$

for any $x \in[a, b]$.

Proof. We use the identity obtained in [9]

$$
f(a)(x-a)+f(b)(b-x)-\int_{a}^{b} f(t) d t=\int_{a}^{b}(t-x) d f(t)
$$

which holds for any Riemann integrable function $f:[a, b] \rightarrow \mathbb{R}$. This can be easily proved integrating by parts in the second integral.

Now, if $f$ is of bounded variation on $[a, b]$, then on applying the first inequality in (2.1) we deduce that:

$$
\begin{aligned}
\left|\int_{a}^{b}(t-x) d f(t)\right| & \leq \int_{a}^{b}|t-x| d\left(\bigvee_{a}^{t}(f)\right) \\
& =\int_{a}^{x}(x-t) d\left(\bigvee_{a}^{t}(f)\right)+\int_{x}^{b}(t-x) d\left(\bigvee_{a}^{t}(f)\right)
\end{aligned}
$$

for any $x \in[a, b]$.

Integrating by parts in the Riemann-Stieltjes integral we have

$$
\begin{aligned}
\int_{a}^{x}(x-t) d\left(\bigvee_{a}^{t}(f)\right) & =\left.(x-t)\left(\bigvee_{a}^{t}(f)\right)\right|_{a} ^{x}+\int_{a}^{x}\left(\bigvee_{a}^{t}(f)\right) d t \\
& =\int_{a}^{x}\left(\bigvee_{a}^{t}(f)\right) d t
\end{aligned}
$$


and

$$
\begin{aligned}
\int_{x}^{b}(t-x) d\left(\bigvee_{a}^{t}(f)\right) & =\left.(t-x)\left(\bigvee_{a}^{t}(f)\right)\right|_{x} ^{b}-\int_{x}^{b}\left(\bigvee_{a}^{t}(f)\right) d t \\
& =(b-x)\left(\bigvee_{a}^{b}(f)\right)-\int_{x}^{b}\left(\bigvee_{a}^{t}(f)\right) d t \\
& =\int_{x}^{b}\left(\bigvee_{a}^{b}(f)-\bigvee_{a}^{t}(f)\right) d t=\int_{x}^{b}\left(\bigvee_{t}^{b}(f)\right) d t
\end{aligned}
$$

for any $x \in[a, b]$.

Making use of (2.5)-(2.7) we get the first inequality in (2.3).

Since $\bigvee_{\mathrm{a}}$ is monotonic nondecreasing on $[\mathrm{a}, \mathrm{b}]$ while $\bigvee^{\mathrm{b}}$. is nonincreasing in the same interval, we have

$$
\int_{a}^{x}\left(\bigvee_{a}^{t}(f)\right) d t \leq(x-a) \bigvee_{a}^{x}(f) \text { and } \int_{x}^{b}\left(\bigvee_{t}^{b}(f)\right) d t \leq(b-x) \bigvee_{x}^{b}(f)
$$

for any $x \in[a, b]$, which gives the second inequality in (2.3).

Using the properties of the maximum, we have

$$
\begin{aligned}
& (x-a) \bigvee_{a}^{x}(f)+(b-x) \bigvee_{x}^{b}(f) \\
& \leq\left\{\begin{array}{l}
\max \{x-a, b-x\} \bigvee_{a}^{b}(f) \\
\max \left\{\bigvee_{a}^{x}(f), \bigvee_{x}^{b}(f)\right\}(b-a)
\end{array}\right. \\
& =\left\{\begin{array}{l}
{\left[\frac{1}{2}(b-a)+\left|x-\frac{a+b}{2}\right|\right] \bigvee_{a}^{b}(f)} \\
{\left[\frac{1}{2} \bigvee_{a}^{b}(f)+\frac{1}{2}\left|\bigvee_{a}^{x}(f)-\bigvee_{x}^{b}(f)\right|\right](b-a)}
\end{array}\right.
\end{aligned}
$$

for any $x \in[a, b]$, and the proof is complete.

An important particular case is where $x=\frac{a+b}{2}$, giving:

Corollary 2.3. Let $\mathrm{f}:[\mathrm{a}, \mathrm{b}] \rightarrow \mathbb{C}$ be a function of bounded variation on $[\mathrm{a}, \mathrm{b}]$. Then

$$
\begin{aligned}
& \left|\int_{a}^{b} f(t) d t-\frac{f(a)+f(b)}{2}(b-a)\right| \\
& \leq \int_{a}^{\frac{a+b}{2}}\left(\bigvee_{a}^{t}(f)\right) d t+\int_{\frac{a+b}{2}}^{b}\left(\bigvee_{t}^{b}(f)\right) d t \leq \frac{1}{2}(b-a) \bigvee_{a}^{b}(f) .
\end{aligned}
$$

The first inequality in (2.8) is sharp. The constant $\frac{1}{2}$ in the second inequality is best possible. 
Proof. We must prove only the sharpness of the first inequality in (2.8) and the best constant.

Consider the function $f:[a, b] \rightarrow \mathbb{R}$ given by

$$
f(t):=\left\{\begin{array}{cc}
1, & t=a \\
0, & t \in(a, b) \\
1, & t=b
\end{array}\right.
$$

We observe that $f$ is of bounded variation and the CVF is given by

$$
\bigvee_{a}^{t}(f)=\left\{\begin{array}{cc}
0, & t=a \\
1, & t \in(a, b) \\
2, & t=b
\end{array}\right.
$$

If we replace this function in (2.8) and perform the calculation, we get the same quantity $b-a$ in all three terms.

Corollary 2.4. Let $\mathrm{f}:[\mathrm{a}, \mathrm{b}] \rightarrow \mathbb{C}$ be a function of bounded variation on $[\mathrm{a}, \mathrm{b}]$. If $\mathrm{p} \in(\mathrm{a}, \mathrm{b})$ is a median point in variation, namely $\bigvee_{\mathrm{a}}^{\mathrm{p}}(\mathrm{f})=\bigvee_{\mathrm{p}}^{\mathrm{b}}(\mathrm{f})$, then we have the inequality

$$
\begin{aligned}
& \left|\int_{a}^{b} f(t) d t-[f(a)(p-a)+f(b)(b-p)]\right| \\
& \leq \int_{a}^{p}\left(\bigvee_{a}^{t}(f)\right) d t+\int_{p}^{b}\left(\bigvee_{t}^{b}(f)\right) d t \leq \frac{1}{2}(b-a) \bigvee_{a}^{b}(f) .
\end{aligned}
$$

The first inequality in (2.3) is useful when some properties for the CVF are available, like for instance below:

Corollary 2.5. Let $\mathrm{f}:[\mathrm{a}, \mathrm{b}] \rightarrow \mathbb{C}$ be a function of bounded variation on $[\mathrm{a}, \mathrm{b}]$. If there exists the constants $\mathrm{L}_{\mathrm{a}}, \mathrm{L}_{\mathrm{b}}>0$ and $\alpha, \beta>-1$ so that

$$
\bigvee_{a}^{t}(f) \leq L_{a}(t-a)^{\alpha} \text { for any } t \in(a, b]
$$

and

$$
\bigvee_{\mathrm{t}}^{\mathrm{b}}(\mathrm{f}) \leq \mathrm{L}_{\mathrm{b}}(\mathrm{b}-\mathrm{t})^{\beta} \text { for any } \mathrm{t} \in[\mathrm{a}, \mathrm{b}) \text {, }
$$

then

$$
\begin{aligned}
& \left|\int_{a}^{b} f(t) d t-[f(a)(x-a)+f(b)(b-x)]\right| \\
& \leq \frac{1}{\alpha+1} L_{a}(x-a)^{\alpha+1}+\frac{1}{\beta+1} L_{b}(b-t)^{\beta+1}
\end{aligned}
$$

for any $x \in[a, b]$. 
The inequality (2.12) follows by integrating the inequalities (2.10) and (2.12) via (2.3).

Corollary 2.6. Let $\mathrm{f}:[\mathrm{a}, \mathrm{b}] \rightarrow \mathbb{C}$ be a function of bounded variation on $[\mathrm{a}, \mathrm{b}]$. If there exists the constant $\mathrm{L}>0$ so that

$$
\bigvee_{\mathrm{a}}^{\mathrm{t}}(\mathrm{f}) \leq \mathrm{L}(\mathrm{t}-\mathrm{a}) \text { and } \bigvee_{\mathrm{t}}^{\mathrm{b}}(\mathrm{f}) \leq \mathrm{L}(\mathrm{b}-\mathrm{t}) \text { for any } \mathrm{t} \in[\mathrm{a}, \mathrm{b}]
$$

then

$$
\begin{aligned}
& \left|\int_{a}^{b} f(t) d t-[f(a)(x-a)+f(b)(b-x)]\right| \\
& \leq\left[\frac{1}{4}+\left(\frac{x-\frac{a+b}{2}}{b-a}\right)\right] L(b-a)^{2}
\end{aligned}
$$

for any $\mathrm{x} \in[\mathrm{a}, \mathrm{b}]$. The constant $\frac{1}{4}$ is best possible.

In particular, we have

$$
\left|\int_{a}^{b} f(t) d t-\frac{f(a)+f(b)}{2}(b-a)\right| \leq \frac{1}{4} L(b-a)^{2} .
$$

The constant $\frac{1}{4}$ is best possible.

Proof. First, we notice that if $h:[a, b] \rightarrow \mathbb{C}$ is of bounded variation, then $|h|:[a, b] \rightarrow[0, \infty)$ is of bounded variation and

$$
\bigvee_{\mathrm{a}}^{\mathrm{b}}(|\mathrm{h}|) \leq \bigvee_{\mathrm{a}}^{\mathrm{b}}(\mathrm{h})
$$

Indeed, by the continuity property of the modulus, we have that

$$
\sum_{j=0}^{n-1}|| h\left(t_{j+1}\right)|-| h\left(t_{j}\right)|| \leq \sum_{j=0}^{n-1}\left|h\left(t_{j+1}\right)-h\left(t_{j}\right)\right|
$$

for any division $a=t_{0}<t_{1}<\ldots<t_{n-1}<t_{n}=b$, which, by taking the supremum over all divisions of $[a, b]$, produces the desired inequality (2.16).

If we consider the function $f_{0}:[a, b] \rightarrow \mathbb{R}, f_{0}(s):=\left|s-\frac{a+b}{2}\right|$ then, by denoting with e the identity function on $[a, b]$, i.e. $\mathbf{e}(t)=t, t \in[a, b]$, we have

$$
\bigvee_{a}^{t}\left(\left|e-\frac{a+b}{2}\right|\right) \leq \bigvee_{a}^{t}\left(e-\frac{a+b}{2}\right)=\bigvee_{a}^{t}(e)=t-a
$$

for any $t \in[a, b]$.

Similarly,

$$
\bigvee_{t}^{b}\left(\left|e-\frac{a+b}{2}\right|\right) \leq b-t
$$


for any $t \in[a, b]$, showing that $f_{0}$ satisfies the condition (2.13) with $L=1$.

Since

$$
\frac{f_{0}(a)+f_{0}(b)}{2}=b-a, \int_{a}^{b} f_{0}(t) d t=\frac{1}{4}(b-a)^{2},
$$

then we get in both sides of (2.15) the same quantity $\frac{1}{4}(b-a)^{2}$. This proves the sharpness of the constant $\frac{1}{4}$ in (2.15) and therefore in (2.14).

Remark 2.7. The inequalities 2.14) and (2.15) are known in the case of Lipschitzian functions with the constant $\mathrm{L}>0$. We obtained them here under weaker conditions for the function $\mathrm{f}$. This show that the refinement in terms of the CVF for the trapezoid inequality (2.3) is also useful to extend known results to larger classes of functions.

The following similar result also holds:

Theorem 2.8. Let $\mathrm{f}:[\mathrm{a}, \mathrm{b}] \rightarrow \mathbb{C}$ be a function of bounded variation on $[\mathrm{a}, \mathrm{b}]$. Then

$$
\begin{aligned}
& \left|\int_{a}^{b} f(t) d t-[f(a)(x-a)+f(b)(b-x)]\right| \\
& \leq \int_{a}^{x}\left(\bigvee_{a}^{t}(|f-f(a)|)\right) d t+\int_{x}^{b}\left(\bigvee_{t}^{b}(|f(b)-f|)\right) d t \\
& \leq(x-a) \bigvee_{a}^{b}(|f-f(a)|)+(b-x) \bigvee_{x}^{x}(|f(b)-f|) \\
& \leq(x-a) \bigvee_{a}^{x}(f)+(b-x) \bigvee_{x}(f) \\
& \leq\left\{\begin{array}{c}
{\left[\frac{1}{2}(b-a)+\left|x-\frac{a+b}{2}\right|\right] \bigvee_{a}^{b}(f),} \\
{\left[\frac{1}{2} \bigvee_{a}^{b}(f)+\frac{1}{2}\left|\bigvee_{a}^{x}(f)-\bigvee_{x}^{b}(f)\right|\right](b-a),}
\end{array}\right.
\end{aligned}
$$

for any $x \in[a, b]$.

Proof. We observe that

$$
\begin{aligned}
& \left|\int_{a}^{b} f(t) d t-[f(a)(x-a)+f(b)(b-x)]\right| \\
& =\left|\int_{a}^{x}(f(a)-f(t)) d t+\int_{x}^{b}(f(b)-f(t)) d t\right| \\
& \leq \int_{a}^{x}|f(a)-f(t)| d t+\int_{x}^{b}|f(b)-f(t)| d t
\end{aligned}
$$

for any $x \in[a, b]$. 
For a given $x \in(a, b)$, define the function $g:[a, b] \rightarrow[0, \infty)$ given by

$$
g(t):= \begin{cases}|f(t)-f(a)|, & t \in[a, x] \\ |f(b)-f(t)|, & t \in(x, b] .\end{cases}
$$

We observe that $g$ is of bounded variation on the intervals $[a, x]$ and $[x, b], g(a)=g(b)=0$. Moreover,

$$
\int_{a}^{b} g(t) d t=\int_{a}^{x}|f(a)-f(t)| d t+\int_{x}^{b}|f(b)-f(t)| d t
$$

for any $x \in(a, b)$.

Since $\mathrm{g}$ is nonnegative, observe also that

$$
\int_{a}^{b} g(t) d t=\left|\int_{a}^{b} g(t) d t-(b-x) g(b)-(x-a) g(a)\right|
$$

for any $x \in(a, b)$.

Now, if we apply Theorem 2.2 for the function $g$ (we should notice that the Theorem 2.2 also holds if we assume the involved function is of bounded variation on the portions $[a, x]$ and $[x, b]$ ), then we get

$$
\begin{aligned}
& \left|\int_{a}^{b} g(t) d t-(b-x) g(b)-(x-a) g(a)\right| \\
& \leq \int_{a}^{x}\left(\bigvee_{a}^{t}(g)\right) d t+\int_{x}^{b}\left(\bigvee_{t}^{b}(g)\right) d t \\
& \leq(x-a) \bigvee_{a}^{x}(g)+(b-x) \bigvee_{x}^{b}(g) .
\end{aligned}
$$

Since

$$
\begin{aligned}
& \int_{a}^{x}\left(\bigvee_{a}^{t}(g)\right) d t+\int_{x}^{b}\left(\bigvee_{t}^{b}(g)\right) d t \\
& =\int_{a}^{x}\left(\bigvee_{a}^{t}(|f-f(a)|)\right) d t+\int_{x}^{b}\left(\bigvee_{t}^{b}(|f(b)-f|)\right) d t
\end{aligned}
$$

and

$$
\begin{aligned}
& (x-a) \bigvee_{a}^{x}(g)+(b-x) \bigvee_{x}^{b}(g) \\
& =(x-a) \bigvee_{a}^{x}(|f-f(a)|)+(b-x) \bigvee_{x}^{b}(|f(b)-f|)
\end{aligned}
$$


then we get from (2.18) and (2.19) the first two inequalities in (2.17).

For the last part, we observe that

$$
\bigvee_{a}^{x}(|f-f(a)|) \leq \bigvee_{a}^{x}(f-f(a))=\bigvee_{a}^{x}(f)
$$

and

$$
\bigvee_{x}^{b}(|f(b)-f|) \leq \bigvee_{x}^{b}(f(b)-f)=\bigvee_{x}^{b}(f)
$$

for any $x \in(a, b)$.

The proof is complete.

Corollary 2.9. Let $\mathrm{f}:[\mathrm{a}, \mathrm{b}] \rightarrow \mathbb{C}$ be a function of bounded variation on $[\mathrm{a}, \mathrm{b}]$. Then

$$
\begin{aligned}
& \left|\int_{a}^{b} f(t) d t-\frac{f(a)+f(b)}{2}(b-a)\right| \\
& \leq \int_{a}^{\frac{a+b}{2}}\left(\bigvee_{a}^{t}(|f-f(a)|)\right) d t+\int_{\frac{a+b}{2}}^{b}\left(\bigvee_{t}^{b}(|f(b)-f|)\right) d t \\
& \leq \frac{1}{2}(b-a)\left[\bigvee_{a}^{\frac{a+b}{2}}(|f-f(a)|)+\bigvee_{\frac{a+b}{2}}^{b}(|f(b)-f|)\right] \\
& \leq \frac{1}{2}(b-a) \bigvee_{a}^{b}(f) .
\end{aligned}
$$

The inequalities in (2.20) are sharp.

Proof. We must prove only the sharpness of the inequalities.

Consider the function of bounded variation $f:[a, b] \rightarrow \mathbb{R}$ given by

$$
f(t):=\left\{\begin{array}{lc}
1, & t=a \\
0, & t \in(a, b) \\
1, & t=b
\end{array}\right.
$$

Observe that

$$
|f(t)-f(a)|=|f(b)-f(t)|=\left\{\begin{array}{cc}
0, & t=a \\
1, & t \in(a, b) \\
0, & t=b
\end{array}\right.
$$

Then

$$
\bigvee_{a}^{t}(|f-f(a)|)=1, t \in\left[a, \frac{a+b}{2}\right]
$$


and

$$
\bigvee_{t}^{b}(|f(b)-f|)=1, t \in\left[\frac{a+b}{2}, b\right]
$$

Replacing these values in (2.20) and performing the calculations we obtain the same quantity $b-a$ in all terms. This proves the sharpness of all inequalities in (2.20).

\section{Applications for Selfadjoint Operators}

We denote by $\mathcal{B}(\mathrm{H})$ the Banach algebra of all bounded linear operators on a complex Hilbert space $(H ;\langle\cdot, \cdot\rangle)$. Let $A \in \mathcal{B}(H)$ be selfadjoint and let $\varphi_{\lambda}$ be defined for all $\lambda \in \mathbb{R}$ as follows

$$
\varphi_{\lambda}(s):=\left\{\begin{array}{l}
1, \text { for }-\infty<s \leq \lambda, \\
0, \text { for } \lambda<s<+\infty .
\end{array}\right.
$$

Then for every $\lambda \in \mathbb{R}$ the operator

$$
E_{\lambda}:=\varphi_{\lambda}(A)
$$

is a projection which reduces $A$.

The properties of these projections are collected in the following fundamental result concerning the spectral representation of bounded selfadjoint operators in Hilbert spaces, see for instance [21, p. 256]:

Theorem 3.1 (Spectral Representation Theorem). Let $A$ be a bounded selfadjoint operator on the Hilbert space $\mathrm{H}$ and let $\mathrm{m}=\min \{\lambda \mid \lambda \in \operatorname{Sp}(A)\}=: \min \operatorname{Sp}(A)$ and $M=\max \{\lambda \mid \lambda \in \operatorname{Sp}(A)\}$ $=: \max S p(A)$. Then there exists a family of projections $\left\{E_{\lambda}\right\}_{\lambda \in \mathbb{R}}$, called the spectral family of $A$, with the following properties

a) $\mathrm{E}_{\lambda} \leq \mathrm{E}_{\lambda^{\prime}}$ for $\lambda \leq \lambda^{\prime}$

b) $\mathrm{E}_{\mathrm{m}-0}=0, \mathrm{E}_{\mathrm{M}}=\mathrm{I}$ and $\mathrm{E}_{\lambda+0}=\mathrm{E}_{\lambda}$ for all $\lambda \in \mathbb{R}$;

c) We have the representation

$$
A=\int_{m-0}^{M} \lambda d E_{\lambda}
$$

More generally, for every continuous complex-valued function $\varphi$ defined on $\mathbb{R}$ and for every $\varepsilon>0$ there exists a $\delta>0$ such that

$$
\left\|\varphi(A)-\sum_{k=1}^{n} \varphi\left(\lambda_{k}^{\prime}\right)\left[E_{\lambda_{k}}-E_{\lambda_{k-1}}\right]\right\| \leq \varepsilon
$$


whenever

$$
\left\{\begin{array}{l}
\lambda_{0}<m=\lambda_{1}<\ldots<\lambda_{n-1}<\lambda_{n}=M \\
\lambda_{k}-\lambda_{k-1} \leq \delta \text { for } 1 \leq k \leq n \\
\lambda_{k}^{\prime} \in\left[\lambda_{k-1}, \lambda_{k}\right] \text { for } 1 \leq k \leq n
\end{array}\right.
$$

this means that

$$
\varphi(A)=\int_{m-0}^{M} \varphi(\lambda) d E_{\lambda},
$$

where the integral is of Riemann-Stieltjes type.

Corollary 3.2. With the assumptions of Theorem 3.1 for $\mathrm{A}, \mathrm{E}_{\lambda}$ and $\varphi$ we have the representations

$$
\varphi(A) x=\int_{m-0}^{M} \varphi(\lambda) \mathrm{dE}_{\lambda} x \text { for all } x \in \mathrm{H}
$$

and

$$
\langle\varphi(A) x, y\rangle=\int_{m-0}^{M} \varphi(\lambda) d\left\langle E_{\lambda} x, y\right\rangle \text { for all } x, y \in H .
$$

In particular,

$$
\langle\varphi(A) \chi, x\rangle=\int_{m-0}^{M} \varphi(\lambda) d\left\langle E_{\lambda} x, x\right\rangle \text { for all } x \in H .
$$

Moreover, we have the equality

$$
\|\varphi(A) x\|^{2}=\int_{m-0}^{M}|\varphi(\lambda)|^{2} \mathrm{~d}\left\|\mathrm{E}_{\lambda} x\right\|^{2} \quad \text { for all } x \in \mathrm{H} .
$$

We need the following result that provides an upper bound for the total variation of the function $\mathbb{R} \ni \lambda \mapsto\left\langle E_{\lambda} x, y\right\rangle \in \mathbb{C}$ on an interval $[\alpha, \beta]$, see [16. For the sake of completeness, we give here a short proof.

Lemma 3.3. Let $\left\{\mathrm{E}_{\lambda}\right\}_{\lambda \in \mathbb{R}}$ be the spectral family of the bounded selfadjoint operator A. Then for any $\mathrm{x}, \mathrm{y} \in \mathrm{H}$ and $\alpha<\beta$ we have the inequality

$$
\left[\bigvee_{\alpha}^{\beta}\left(\left\langle E_{(\cdot)} x, y\right\rangle\right)\right]^{2} \leq\left\langle\left(E_{\beta}-E_{\alpha}\right) x, x\right\rangle\left\langle\left(E_{\beta}-E_{\alpha}\right) y, y\right\rangle,
$$

where $\bigvee_{\alpha}^{\beta}\left(\left\langle\mathrm{E}_{(\cdot)} x, y\right\rangle\right)$ denotes the total variation of the function $\left\langle\mathrm{E}_{(\cdot)} x, y\right\rangle$ on $[\alpha, \beta]$.

Proof. If $\mathrm{P}$ is a positive selfadjoint operator on $\mathrm{H}$, i.e., $\langle\mathrm{P} x, x\rangle \geq 0$ for any $x \in \mathrm{H}$, then the following inequality is a generalization of the Schwarz inequality in $\mathrm{H}$

$$
|\langle P x, y\rangle|^{2} \leq\langle P x, x\rangle\langle P y, y\rangle
$$


for any $x, y \in H$.

Now, if $d: \alpha=t_{0}<t_{1}<\ldots<t_{n-1}<t_{n}=\beta$ is an arbitrary partition of the interval $[\alpha, \beta]$, then we have by Schwarz's inequality for positive operators (3.5) that

$$
\begin{aligned}
& \bigvee_{\alpha}^{\beta}\left(\left\langle E_{(\cdot)} x, y\right\rangle\right) \\
& =\sup _{d}\left\{\sum_{i=0}^{n-1}\left|\left\langle\left(E_{t_{i+1}}-E_{t_{i}}\right) x, y\right\rangle\right|\right\} \\
& \leq \sup _{d}\left\{\sum_{i=0}^{n-1}\left[\left\langle\left(E_{t_{i+1}}-E_{t_{i}}\right) x, x\right\rangle^{1 / 2}\left\langle\left(E_{t_{i+1}}-E_{t_{i}}\right) y, y\right\rangle^{1 / 2}\right]\right\}:=B .
\end{aligned}
$$

By the Cauchy-Bunyakovsky-Schwarz inequality for sequences of real numbers we also have that

$$
\begin{aligned}
& \sum_{i=0}^{n-1}\left[\left\langle\left(E_{t_{i+1}}-E_{t_{i}}\right) x, x\right\rangle^{1 / 2}\left\langle\left(E_{t_{i+1}}-E_{t_{i}}\right) y, y\right\rangle^{1 / 2}\right] \\
& \leq\left[\sum_{i=0}^{n-1}\left\langle\left(E_{t_{i+1}}-E_{t_{i}}\right) x, x\right\rangle\right]^{1 / 2}\left[\sum_{i=0}^{n-1}\left\langle\left(E_{t_{i+1}}-E_{t_{i}}\right) y, y\right\rangle\right]^{1 / 2} \\
& =\left[\left\langle\left(E_{\beta}-E_{\alpha}\right) x, x\right\rangle\right]^{1 / 2}\left[\left\langle\left(E_{\beta}-E_{\alpha}\right) y, y\right\rangle\right]^{1 / 2}
\end{aligned}
$$

for any $x, y \in H$. Taking the supremum over $d$ in (3.7) we get

$$
B \leq\left[\left\langle\left(E_{\beta}-E_{\alpha}\right) x, x\right\rangle\right]^{1 / 2}\left[\left\langle\left(E_{\beta}-E_{\alpha}\right) y, y\right\rangle\right]^{1 / 2}
$$

for any $x, y \in \mathrm{H}$ which together with (3.6) produce the desired result (3.4).

Remark 3.4. For $\alpha=m-\varepsilon$ with $\varepsilon>0$ and $\beta=M$ we get from (3.4) the inequality

$$
\bigvee_{m-\varepsilon}^{M}\left(\left\langle E_{(\cdot)} x, y\right\rangle\right) \leq\left\langle\left(I-E_{m-\varepsilon}\right) x, x\right\rangle^{1 / 2}\left\langle\left(I-E_{m-\varepsilon}\right) y, y\right\rangle^{1 / 2}
$$

for any $\mathrm{x}, \mathrm{y} \in \mathrm{H}$.

This implies, for any $\mathrm{x}, \mathrm{y} \in \mathrm{H}$, that

$$
\bigvee_{m-0}^{M}\left(\left\langle E_{(\cdot)} x, y\right\rangle\right) \leq\|x\|\|y\|,
$$

where $\bigvee_{m-0}^{M}\left(\left\langle E_{(\cdot)} x, y\right\rangle\right)$ denotes the limit $\lim _{\mathcal{\varepsilon} \rightarrow 0+}\left[\bigvee_{m-\varepsilon}^{M}\left(\left\langle E_{(\cdot)} x, y\right\rangle\right)\right]$.

The following result holds: 
Theorem 3.5. Let $\mathrm{A}$ be a bounded selfadjoint operator on the Hilbert space $\mathrm{H}$ and let $\mathrm{m}=$ $\min \{\lambda \mid \lambda \in \operatorname{Sp}(A)\}=: \min S p(A)$ and $M=\max \{\lambda \mid \lambda \in \operatorname{Sp}(A)\}=: \max \operatorname{Sp}(A)$. If $\left\{E_{\lambda}\right\}_{\lambda \in \mathbb{R}}$ is the spectral family of the bounded selfadjoint operator $A$, then for any $v \in[m, M]$ we have

$$
\begin{aligned}
& |\langle(A-v I) x, y\rangle| \\
& \leq \int_{m-0}^{v}\left\langle E_{t} x, x\right\rangle^{1 / 2}\left\langle E_{t} y, y\right\rangle^{1 / 2} d t \\
& +\int_{v}^{M}\left\langle\left(I-E_{t}\right) x, x\right\rangle^{1 / 2}\left\langle\left(I-E_{t}\right) y, y\right\rangle^{1 / 2} d t \\
& \leq(v-m)\left\langle E_{v} x, x\right\rangle^{1 / 2}\left\langle E_{v} y, y\right\rangle^{1 / 2} \\
& +(M-v)\left\langle\left(I-E_{v}\right) x, x\right\rangle^{1 / 2}\left\langle\left(I-E_{v}\right) y, y\right\rangle^{1 / 2} \\
& \leq\left[\frac{1}{2}(M-m)+\left|v-\frac{a+b}{2}\right|\right] \\
& \times\left[\left\langle E_{v} x, x\right\rangle^{1 / 2}\left\langle E_{v} y, y\right\rangle^{1 / 2}+\left\langle\left(I-E_{v}\right) x, x\right\rangle^{1 / 2}\left\langle\left(I-E_{v}\right) y, y\right\rangle^{1 / 2}\right] \\
& \leq\left[\frac{1}{2}(M-m)+\left|v-\frac{a+b}{2}\right|\right]\|x\|\|y\|
\end{aligned}
$$

for any $\mathrm{x}, \mathrm{y} \in \mathrm{H}$.

In particular, we have

$$
\begin{aligned}
& \left|\left\langle\left(A-\frac{m+M}{2} I\right) x, y\right\rangle\right| \\
& \leq \int_{\frac{m}{2}-0}^{\frac{m+M}{2}}\left\langle E_{t} x, x\right\rangle^{1 / 2}\left\langle E_{t} y, y\right\rangle^{1 / 2} d t \\
& +\int_{\frac{m+M}{2}}^{M}\left\langle\left(I-E_{t}\right) x, x\right\rangle^{1 / 2}\left\langle\left(I-E_{t}\right) y, y\right\rangle^{1 / 2} d t \\
& \leq \frac{1}{2}(M-m)\left[\left\langle E_{\frac{m+M}{2}} x, x\right\rangle^{1 / 2}\left\langle E_{\frac{m+M}{2}} y, y\right\rangle^{1 / 2}\right. \\
& \left.+\left\langle\left(I-E_{\frac{m+M}{2}}\right) x, x\right\rangle^{1 / 2}\left\langle\left(I-E_{\frac{m+M}{2}}\right) y, y\right\rangle^{1 / 2}\right] \\
& \leq \frac{1}{2}(M-m)\|x\|\|y\|
\end{aligned}
$$

for any $\mathrm{x}, \mathrm{y} \in \mathrm{H}$.

Proof. Utilising the representation in (3.3) we have

$$
|\langle(A-v I) x, y\rangle|=\left|\int_{m-0}^{M}(t-v) d\left\langle E_{t} x, y\right\rangle\right|
$$

for any $v \in[m, M]$ and for any $x, y \in H$. 
For $\varepsilon>0$, by utilizing an argument similar to the one in Theorem 2.2 we have

$$
\begin{aligned}
& \left|\int_{m-\varepsilon}^{M}(t-v) d\left\langle E_{t} x, y\right\rangle\right| \\
& \leq\left|\int_{m-\varepsilon}^{M}\right| t-v\left|d\left(\bigvee_{a}^{t}\left\langle E_{t} x, y\right\rangle\right)\right| \\
& \leq \int_{m-\varepsilon}^{v}\left(\bigvee_{a}^{t}\left\langle E_{t} x, y\right\rangle\right) d t+\int_{v}^{M}\left(\bigvee_{t}^{M}\left\langle E_{t} x, y\right\rangle\right) d t
\end{aligned}
$$

for any $v \in[m, M]$ and for any $x, y \in H$.

From Lemma 3.3 we have

$$
\begin{aligned}
& \int_{m-\varepsilon}^{v}\left(\bigvee_{a}^{t}\left\langle E_{t} x, y\right\rangle\right) d t \\
& \leq \int_{m-\varepsilon}^{v}\left\langle\left(E_{t}-E_{m-\varepsilon}\right) x, x\right\rangle^{1 / 2}\left\langle\left(E_{t}-E_{m-\varepsilon}\right) y, y\right\rangle^{1 / 2} d t
\end{aligned}
$$

and

$$
\begin{aligned}
& \int_{v}^{M}\left(\bigvee_{t}^{M}\left\langle E_{t} x, y\right\rangle\right) d t \\
& \leq \int_{v}^{M}\left\langle\left(E_{M}-E_{t}\right) x, x\right\rangle^{1 / 2}\left\langle\left(E_{M}-E_{t}\right) y, y\right\rangle^{1 / 2} d t \\
& =\int_{v}^{M}\left\langle\left(I-E_{t}\right) x, x\right\rangle^{1 / 2}\left\langle\left(I-E_{t}\right) y, y\right\rangle^{1 / 2} d t
\end{aligned}
$$

for any $v \in[m, M]$ and for any $x, y \in H$.

Therefore,

$$
\begin{aligned}
& \left|\int_{m-\varepsilon}^{M}(t-v) d\left\langle E_{t} x, y\right\rangle\right| \\
& \leq \int_{m-\varepsilon}^{v}\left\langle\left(E_{t}-E_{m-\varepsilon}\right) x, x\right\rangle^{1 / 2}\left\langle\left(E_{t}-E_{m-\varepsilon}\right) y, y\right\rangle^{1 / 2} d t \\
& +\int_{v}^{M}\left\langle\left(I-E_{t}\right) x, x\right\rangle^{1 / 2}\left\langle\left(I-E_{t}\right) y, y\right\rangle^{1 / 2} d t
\end{aligned}
$$

for any $\varepsilon>0$, for any $v \in[m, M]$ and for any $x, y \in H$. 
Taking the limit over $\varepsilon \rightarrow 0+$ and since $\mathrm{E}_{\mathrm{m}-0}=0$, we get

$$
\begin{aligned}
& \left|\int_{m-0}^{M}(t-v) d\left\langle E_{t} x, y\right\rangle\right| \\
& \leq \int_{m-0}^{v}\left\langle E_{t} x, x\right\rangle^{1 / 2}\left\langle E_{t} y, y\right\rangle^{1 / 2} d t \\
& +\int_{v}^{M}\left\langle\left(I-E_{t}\right) x, x\right\rangle^{1 / 2}\left\langle\left(I-E_{t}\right) y, y\right\rangle^{1 / 2} d t
\end{aligned}
$$

for any $v \in[m, M]$ and for any $x, y \in H$, which proves the first inequality in (3.13).

The rest is easy to see and we omit the details.

Received: March 2015. Accepted: May 2015.

\section{References}

[1] N. S. Barnett and S.S. Dragomir, A perturbed trapezoid inequality in terms of the third derivative and applications. Inequality theory and applications. Vol. 5, 1-11, Nova Sci. Publ., New York, 2007.

[2] N. S. Barnett and S.S. Dragomir, Perturbed version of a general trapezoid inequality. Inequality theory and applications. Vol. 3, 1-12, Nova Sci. Publ., Hauppauge, NY, 2003.

[3] N. S. Barnett and S.S. Dragomir, A perturbed trapezoid inequality in terms of the fourth derivative. Korean J. Comput. Appl. Math. 9 (2002), no. 1, 45-60.

[4] N. S. Barnett, S.S. Dragomir and I. Gomm, A companion for the Ostrowski and the generalised trapezoid inequalities. Math. Comput. Modelling 50 (2009), no. 1-2, 179-187.

[5] N. S. Barnett, W.-S. Cheung, S.S. Dragomir and A. Sofo, Ostrowski and trapezoid type inequalities for the Stieltjes integral with Lipschitzian integrands or integrators. Comput. Math. Appl. 57 (2009), no. 2, 195-201.

[6] P. Cerone, W.-S. Cheung and S.S. Dragomir, On Ostrowski type inequalities for Stieltjes integrals with absolutely continuous integrands and integrators of bounded variation. Comput. Math. Appl. 54 (2007), No. 2, 183-191.

[7] P. Cerone and S.S. Dragomir, Midpoint-type rules from an inequalities point of view. Handbook of analytic-computational methods in applied mathematics, 135-200, Chapman \& Hall/CRC, Boca Raton, FL, 2000.

[8] P. Cerone and S.S. Dragomir, Trapezoidal-type rules from an inequalities point of view. Handbook of analytic-computational methods in applied mathematics, 65-134, Chapman \& Hall/CRC, Boca Raton, FL, 2000. 
[9] P. Cerone, S.S. Dragomir and C.E.M. Pearce, A generalised trapezoid inequality for functions of bounded variation, Turk. J. Math., 24 (2000), 147-163.

[10] X.-L. Cheung and J. Sun, A note on the perturbed trapezoid inequality. J. Inequal. Pure Appl. Math. 3 (2002), no. 2, Article 29, 7 pp. (electronic).

[11] S.S. Dragomir, The Ostrowski inequality for mappings of bounded variation, Bull. Austral. Math. Soc., 60 (1999), 495-826.

[12] S.S. Dragomir, On the mid-point quadrature formula for mappings with bounded variation and applications, Kragujevac J. Math., 22 (2000), 13-19.

[13] S.S. Dragomir, On the trapezoid quadrature formula and applications, Kragujevac J. Math., 23 (2001), 25-36.

[14] S.S. Dragomir, Improvements of Ostrowski and generalised trapezoid inequality in terms of the upper and lower bounds of the first derivative. Tamkang J. Math. 34 (2003), no. 3, 213-222.

[15] S.S. Dragomir, Some inequalities of midpoint and trapezoid type for the Riemann-Stieltjes integral. Proceedings of the Third World Congress of Nonlinear Analysts, Part 4 (Catania, 2000). Nonlinear Anal. 47 (2001), no. 4, 2333-2340.

[16] S.S. Dragomir, Some inequalities for continuous functions of selfadjoint operators in Hilbert spaces, Acta Math. Vietnamica, to appear. Preprint RGMIA Res. Rep. Coll. 15(2012), Art. 16. http://rgmia.org/v15.php.

[17] S.S. Dragomir,Y. J. Cho and Y.-H. Kim, On the trapezoid inequality for the Riemann-Stieltjes integral with Hölder continuous integrands and bounded variation integrators. Inequality theory and applications. Vol. 5, 71-79, Nova Sci. Publ., New York, 2007.

[18] S.S. Dragomir and A. McAndrew, On trapezoid inequality via a Grüss type result and applications. Tamkang J. Math. 31 (2000), no. 3, 193-201.

[19] S.S. Dragomir, J. Pečarić and S. Wang, The unified treatment of trapezoid, Simpson, and Ostrowski type inequality for monotonic mappings and applications. Math. Comput. Modelling 31 (2000), no. 6-7, 61-70.

[20] H. Gunawan, A note on Dragomir-McAndrew's trapezoid inequalities. Tamkang J. Math. 33 (2002), no. 3, 241-244.

[21] G. Helmberg, Introduction to Spectral Theory in Hilbert Space, John Wiley \& Sons, Inc. -New York, 1969.

[22] A. I. Kechriniotis and N. D. Assimakis, Generalizations of the trapezoid inequalities based on a new mean value theorem for the remainder in Taylor's formula. J. Inequal. Pure Appl. Math. 7 (2006), no. 3, Article 90, 13 pp. (electronic). 
[23] Z. Liu, Some inequalities of perturbed trapezoid type. J. Inequal. Pure Appl. Math. 7 (2006), no. 2, Article 47, 9 pp.

[24] W.-J. Liu, Q.-L. Xue and J.-W. Dong, New generalization of perturbed trapezoid, mid-point inequalities and applications. Int. J. Pure Appl. Math. 41 (2007), no. 6, 761-768.

[25] P. R. Mercer, Hadamard's inequality and trapezoid rules for the Riemann-Stieltjes integral. J. Math. Anal. Appl. 344 (2008), no. 2, 921-926.

[26] A. McD. Mercer, On perturbed trapezoid inequalities. J. Inequal. Pure Appl. Math. 7 (2006), no. 4, Article 118, 7 pp. (electronic).

[27] B. G. Pachpatte, A note on a trapezoid type integral inequality. Bull. Greek Math. Soc. 49 (2004), 85-90.

[28] B. G. Pachpatte,New inequalities of Ostrowski and trapezoid type for n-time differentiable functions. Bull. Korean Math. Soc. 41 (2004), no. 4, 633-639.

[29] N. Ujević, Error inequalities for a generalized trapezoid rule. Appl. Math. Lett. 19 (2006), no. $1,32-37$.

[30] N. Ujević, On perturbed mid-point and trapezoid inequalities and applications. Kyungpook Math. J. 43 (2003), no. 3, 327-334.

[31] N. Ujević, Perturbed trapezoid and mid-point inequalities and applications. Soochow J. Math. 29 (2003), no. 3, 249-257.

[32] Q. Wu and S. Yang, A note to Ujević's generalization of Ostrowski's inequality. Appl. Math. Lett. 18 (2005), no. 6, 657-665. 